\title{
Diacronie
}

Studi di Storia Contemporanea

$N^{\circ} 17,1 \mid 2014$

Periferie. Cultura, economia, politica

\section{La Seconda guerra mondiale sugli schermi: un'analisi del cinema nel Nordeste brasiliano (1939-1945)}

\section{Andreza Santos Cruz Maynard}

Traduttore: Anita Lucchesi

\section{OpenEdition}

\section{Journals}

\section{Edizione digitale}

URL: http://journals.openedition.org/diacronie/1025

DOI: 10.4000/diacronie.1025

ISSN: 2038-0925

\section{Editore}

Association culturelle Diacronie

Notizia bibliografica digitale

Andreza Santos Cruz Maynard, « La Seconda guerra mondiale sugli schermi: un'analisi del cinema nel Nordeste brasiliano (1939-1945) », Diacronie [Online], № 17, 1 | 2014, documento 4, Messo online il 01 mars 2014, consultato il 30 avril 2019. URL : http://journals.openedition.org/diacronie/1025 ; DOI : 10.4000/diacronie. 1025 


\title{
Diacronie
}

\section{La Seconda guerra mondiale sugli schermi:} una analisi del cinema nel Nordeste brasiliano (1939-1945)

\author{
Andreza SANTOS CRUZ MAYNARD *
}

traduzione di Anita LUCCHESI

L'articolo prende in esame il rapporto tra i cinema della città di Aracaju (capitale dello stato di Sergipe) ed i film proiettati durante la Seconda guerra mondiale. La programmazione dei cinema è cambiata in funzione della produzione nordamericana anche in funzione del posizionamento del Brasile in guerra e nei confronti dei paesi coinvolti nel conflitto. I giornali e le riviste che davano conto delle nuove pellicole delle grandi produttrici nordamericane sono alla base di questa analisi della funzione del film in un periodo in cui i mezzi di comunicazione di massa erano controllati per finalità politiche.

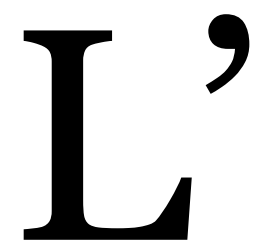

invenzione del cinema ed i primi film incantarono il pubblico in vari paesi: in Brasile si accontentò del consumo dei film stranieri. In virtù della scarsa produzione nazionale nei primi decenni del ventesimo secolo, per non menzionare la differenza della qualità tecnica delle produzioni patrie, i cinema brasiliani furono invasi dai film europei e nordamericani. Durante la Seconda guerra mondiale il Brasile strinse legami politici, economici e culturali con gli Stati Uniti. Questo riavvicinamento aprì ancora più le porte del paese alla produzione cinematografica hollywoodiana.

I grandi studi nordamericani lavoravano in Brasile fin dalla fine degli anni Dieci del Novecento. Grazie agli uffici stabiliti in alcune città, le produzioni nordamericane 
approfittavano della politica della Boa Vizinhança, del "buon vicinato", per riscuotere consenso presso il pubblico brasiliano che, secondo Cristina Menguello², tra gli anni Trenta e Quaranta era il terzo più grande consumatore mondiale dei film prodotti negli Stati Uniti. In questo senso, l'articolo prende in esame il rapporto tra i cinema della città di Aracaju (capitale dello Stato del Sergipe) ed i film proiettati durante gli anni della Seconda guerra mondiale.

I giornali che circolavano nella capitale di Sergipe tra 1939 e 1945 sono state le principali fonti documentali consultate per la realizzazione di questo articolo. Periodici come il «Correio de Aracaju», «Folha da Manhã», «A cruzada», «Sergipe Jornal» e «O Nordeste» venivano utilizzati come mezzi per la circolazione dei programmi giornalieri delle principali sale di proiezione del luogo. Ogni locale era in grado di inviare la lista dei film in proiezione, e assieme a questa, la sinossi o la rassegna stampa del film che arrivava alle sale di proiezione; in alcuni casi facevano in modo che si pubblicassero sui giornali anche delle immagini tratte dai film. Durante la guerra era normale che i film arrivassero in città col treno che veniva da Salvador (capitale dello Stato di Bahia), dove le case produttrici nordamericane avevano i loro uffici.

In quanto mezzo di comunicazione, il cinema era sotto la sorveglianza del DIP (acronimo portoghese per Departemento de Imprensa e Propaganda, Dipartimento per la Stampa e la Propaganda), creato per promuovere l'Estado Novo brasiliano3. I film nazionali ed internazionali che arrivavano ad Aracaju erano perciò in un primo momento valutati dal DIP. Questa misura mirava ad evitare critiche nei confronti del regime politico e la circolazione di notizie favorevoli alla Germania, all'Italia e al Giappone. L'attenzione verso i film proiettati in questo periodo diventava centrale per il governo, dato che il cinema non può essere considerato soltanto una forma d'arte, poiché esso è, «innanzitutto, un mezzo di comunicazione e riproduzione [...] può avere

\footnotetext{
${ }^{1}$ Secondo Maria Lígia Prado Coelho, questa pratica fu caratterizzata dal cambiamento dei pilastri fondamentali della politica estera degli Stati Uniti. Portata avanti dal presidente Roosevelt e dal suo sottosegretario Sumner Welles, consisteva nel rispettare la sovranità nazionale dagli altri paesi del continente. Cfr. PRADO, Maria Ligia Coelho, «Ser ou não ser um bom vizinho: América Latina e Estados Unidos durante a Guerra», in Revista USP São Paulo, 26 3/1995, pp. 52-61, URL:

< http://www.revistas.usp.br/revusp/article/view/28145/29956 > [consultato il 10 marzo 2014].

${ }^{2}$ Cfr. MENGUELLO, Cristina, Poeira de estrelas: o cinema hollywoodiano na mídia brasileira das décadas de 40 e 5o, Campinas (SP), Editora da UNICAMP, 1996.

3 Creato per effetto del Decreto n. 9.915, del 27 dicembre 1939, il Dipartimento per la Stampa e la Propaganda (DIP) aveva il compito di controllare tutta la propaganda e la pubblicità in favore degli Enti pubblici, oltre ad organizzare celebrazioni per il presidente Getulio Vargas. Il DIP era il portavoce dell'Estado Novo. Cfr. ARAÚJO, Rejane, Dipartimento de Imprensa e Propaganda, sub vocem, in ABREU, Alzira Alves de, et al., Dicionário Histórico-Biográfico Brasileiro (Pós 1930), Rev. Amp. Atual, vol. 1, Rio de Janeiro, Editora FGV-CPDOC, 2001.
} 
come obiettivo la semplice divulgazione di dati, senza che ciò abbia nulla a che vedere con l'estetica»4.

Il DIP lavorava in collaborazione con l'Office of Inter American Affairs (OCIAA)5, l'agenzia brasiliana che sviluppava attività anche in Brasile. Ne era capo Rockfeller e lo OCIAA era risponsabile dai rapporti di boa vizinhança degli Stati Uniti con gli altri paesi del continente. Ed il cinema ottenne un trattamento speciale nell'intento di far sì nessun film sembrasse offensivo ai latinoamericani; per questa ragione «[...] vennero censurate scene, furono proibiti certi argomenti, furono suggerite modifiche alle sceneggiature»6. Negli Stati Uniti le case produttrici potevano concorrere liberamente, però all'estero l'azione era legata alla Motion Picture Association of America, che improntava le sue attività sulla dinamiche della politica estera della Casa Bianca7.

Tuttavia gli Stati Uniti non speravano che i brasiliani acquisissero semplicemente e soltanto l'idea che loro erano buoni vicini. Lo sforzo per promuovere la diffusione del film nordamericano non voleva soltanto rafforzare i legami tra i due paesi. Il predecessore di Franklin Delano Roosevelt (1933-1945), Herbert Hoover (1929-1933) aveva già esplicitato che «dove entra il film americano, vendiamo più macchine americane, più cappelli, più giradischi americani» 8 .

Così in Brasile veniva a crearsi un immenso mercato per i film, ma anche per il modo di vivere nordamericano e per i prodotti industrializzati che avrebbero permesso di tramutare in realtà questo "sogno". In questa senso la cultura nordamericana si avvicinò alla quotidianità dei brasiliani, dato che i film prodotti da Hollywood aiutarono a diffondere l'american way of life in tutto il continente.

I cinema erano tra le poche offerte di divertimento su cui poteva contare la populazione di Aracaju, che constava di circa 60.000 persone9. I principali locali erano i cinema Rio Branco, São Francisco, Guarany, Rex e Vitória. Ciascuno di essi disponeva

\footnotetext{
${ }_{4}^{4}$ ROSENFELD, Anatol, Cinema: arte \& indústria, São Paulo, Editora Perspectiva, 2002, p. 33.

5 Il Consiglio di Sicurezza Nazionale degli Stati Uniti creò l'Office for the Coordinator of Commercial and Cultural Relations between the American Republicas, il 16 agosto 1940. Tra il 30 luglio 1941 e il 23 marzo 1945 assunse la denominazione di Office of Coordinator InterAmerican Affairs (OCIAA). L'ente si sarebbe dovuto occupare di coordinare il rapporto economico e culturale tra i paesi americani.

6 PRADO, Maria Ligia Coelho, op. cit., p. 60.

7 Erede dalla prospera ditta di esplorazione petroliera Standard Oil, Nelson Rockfeller era stato direttore generale del MOMA in New York e credeva poter utilizzare l'arte con fini politici. Cf. TOTA, Antonio Pedro, O imperialismo sedutor: a americanização do Brasil na época da segunda guerra, São Paulo, Companhia das Letras, 2000.

8 HOOVER, Herbert, cit. in CALIL, Carlos Augusto, Cinema e Indústria, in XAVIER, Ismail (org.), O cinema no século, Rio de Janeiro, Imago Ed., 1996, pp. 45-70. La citazione si trova a p. 60.

9 Secondo il censimento del 1940, Aracaju aveva in quell'anno 59.031 abitanti. Cfr. IBGE, URL:

< http://www.ibge.gov.br/home/ > [consultato il 24 gennaio 2014].
} 
di una sala di esibizione, però alcuni arrivavano a mille posti. Erano definiti anche "cineteatri" perché, oltre allo schermo, erano provvisiti anche di un palco che permetteva rappresentazioni di gruppi teatrali, musicisti e di altri artisti. Tutti i giorni i "cineteatri” annunciavano i loro programmi, i prezzi, gli sconti speciali e le novità.

I film stranieri non trovavano grande concorrenza tra le produzioni nazionali, rare e di difficile distribuzione. Paulo Emílio Sales Gomes segnala la inespressività del cinema brasiliano durante la Seconda Guerra Mondiale. Il critico spiega che «tra il 1908 e il 1911, la città di Rio conobbe l'età dell'oro del cinema brasiliano, giudizio valido alla luce delle grigie frustrazioni nei decenni successivi» ${ }^{10}$,e che questo accadeva grazie alle iniziative degli stranieri che arrivavano nel paese, dal momento che era necessario investire e sapere adoperare i macchinari. Questo ragione sembra, almeno inizialmente, non aver attirato molti brasiliani verso l'attività cinematografica. In ogni modo il grande pubblico preferiva i film importati.

Sotto l'amministrazione del presidente Getulio Vargas ${ }^{11}$ comparvero le prime leggi che assicuravano la continuità dei «pessimi cinegiornali e che, in una fase posteriore, obbligarono le sale a proiettare un piccola percentuale di film brasiliani con una trama»12. Negli anni Trenta e Quaranta la produzione era stata quasi esclusivamente carioca. A São Paulo, erano stati realizzati alcuni studi cinematografici, ma venne prodotto solo il film Eterna esperança ${ }^{13}$. Secondo Gomes, tra il 1933 e il 1949, la produzione si limitò a questo film nello Stato di São Paulo, ad un altro nello Stato di Minas Gerais e ad un ultimo nel Pernambuco. Nel 1942, anno in cui il Brasile dichiarò guerra alla Germania e all'Italia, la produzione nazionale si limitò a due film e crebbe fino a raggiungere la quota di venti film solo nel 1949. La scarsa circolazione di queste pellicole contribuiva al successo dei film e delle serie che giungevano degli Stati Uniti.

In considerazione del fatto che si trattava di una possibilità di svago ed informazione accessibile, sia per il prezzo dei biglietti che per il linguaggio, il cinema si pose come un veicolo indispensabile per tenere aggiornata la popolazione su quello che accadeva dentro e fuori dalle pellicole.

Il film a colori comparve solo nel 1933, però era ancora una novità nella Aracaju degli anni Quaranta. Alcuni annunci di giornale mettevano in luce questo differenza

${ }^{10}$ GOMES, Paulo Emílio Sales, Cinema: trajetória no subdesenvolvimento, São Paulo, Paz e terra, 1996, p. 11.

${ }^{11}$ Getúlio Dornelles Vargas fu a capo della Rivoluzione del 1930 in Brasile; divenne il $13^{\circ}$ Presidente della Repubblica del paese e rimase al potere fino a 1945. Nel 1937 lo stesso Getulio Vargas attuò un colpo di stato che ha originò un regime centralista e autoritario chiamato Estado Novo, ispirato dai governi dei paesi fascisti europei. L'Estado Novo durò fino a 1945, quando il Brasile entrò in un fase di democratizzazione.

12 GOMES, Paulo Emílio Sales, op. cit., p. 14.

${ }^{13}$ MARTEN, Leo, Eterna esperança, Cinédia, Brasil, 1940. 
nelle proiezioni. Il cinema São Francisco prometteva che il $1^{\circ}$ luglio 1940 sarebbe entrato in programmazione il «film a colori della United "Nada é Sagrado"14»15. Aldilà dei film e delle serie, proiettate per capitoli, i cinema mostravano anche i cinejornais (cinegiornali). La produzione naizonale rimase indietro anche in questo campo, lasciando spazio ai cinegiornali nordamericani.

Il 6 agosto il cinema Guarany annunciò il debutto del film della casa di produzione Metro Parnell Rei sem coroa ${ }^{16}$, «con Clark Gable e Myrna Loy, in una storia di emozione ed amore!» e «nell'ambito della stessa programmazione, il secondo cinegiornale di guerra "A Voz do Mundo" numero 40X76, rapporto speciale sulla guerra: il Belgio devastato, Attacchi aerei, Bombe incendiarie! Battaglie navali nel Mare del Nord, con in scena la Royal Oak, la portaerei inglese»17. Oltre al divertimento, le notizie sul Brasile ed il mondo giustificavano la frequentazione di questi locali.

Già nel 1939 venivano annunciavano film a colori, così come quelli presentati erano «integralmente doppiati in portoghese», caratteristiche importanti che fungevano da invito per il pubblico per recarsi in questi cinema. Il giornale «O Nordeste» annunciava il 13 marzo 1939 la proiezione di un film «in techinicolor! In tre dimensioni! 90 minuti di proiezione. Tutta doppiata in portoghese» ${ }^{8}$.

Queste novità rappresentavano un cambiamento piuttosto significativo nella fruizione che il pubblico aveva del film. Essi si avvicinavano maggiormente alle immagini reali e, al contempo, lasciavano «un senso di irrealtà, un regno di fantasmi impalpabili»19. Come in una gioco di prestigio, il cinema mostrava simulacri di realtà, spesso utilizzando immagini più perfette della realtà. Così il cinema transitava tra reale ed irreale, sciogliendo o confondendo le barriere senza danneggiare lo spettacolo.

Simbolo di modernità e cosmopolitismo, fin dal principio il cinema si rivolse al grande pubblico. Esso si consegnava al bombardamento di stimoli proprio del Ventesimo secolo, che si proponeva in diverse forme: dall'inquinamento visuale degli annunci propagandistici fino agli spettacoli come quello del «giro della morte», nel quale una macchina eseguiva un'evoluzione in aria pima di toccare terra. Ed il cinema si trasformava anche in un divertimento sensazionalistico, dal momento che «la

\footnotetext{
14 WELLMAN, William, Nothing Sacred, United, Stati Uniti, 1937, 77'. Il film è noto in Italia come Nulla sul serio.

15 Correio de Aracaju, 31 luglio 1940, p. 3.

16 STAHL, John M., Parnell, Metro-Goldwyn-Mayer, Stati Uniti, 1937, 118'. Il film è noto in Italia come Parnell.

17 Correio de Aracaju, 6 agosto 1940, p. 2.

18 O Nordeste, 13 marzo 1939, p. 2.

19 GUNNING, Tom, O retrato do corpo humano: a fotografia, os detetives e os primórdios do cinema, in CHARNEY, Leo, SCHWARTZ, Vanessa R. (org.), O cinema e a invenção da vida moderna, São Paulo, Cosac \& Naify, 2004, pp. 33-65. La citazione proviene da p. 35.
} 
modernità aveva inaugurato le scosse sensoriali» ${ }^{20}$. Questo era un modo alla portata di tutti e relativamente democratico di accesso all'arte.

In questo senso, il cinema fu pensato da Walter Benjamin ${ }^{21}$ e Siegfried Kracauer ${ }^{22}$ in relazione al suo rapporto con le masse. Questa peculiare forma d'arte integrava l'industria del divertimento e ha l'obiettivo di soddisfare un grande mercato. Dalla produzione di un film fino alla sua proiezione, una grande quantità di persone era coinvolta nel processo creativo. Tutti erano responsabili dell'opera finale. I costi erano alti e perciò la stessa produzione dei film veniva accompagnata dalla necessità che questi ultimi circolassero e avessero a disposizone locali per l'ospitare il pubblico durante la proiezione. In questi termini, lo sviluppo della pratica cinematografica, classificata da Benjamin come arte, si associata con l'attività industriale fin dai primordi.

Per definire il cinema come arte, Benjamin mise in discussione innanzitutto il significato dell'opera d'arte e la sua condizione nell'era della sua riproducibilità tecnica. Per lui non aveva senso mettere inserire la produzione dei film in un concetto che comprendesse singoli oggetti. Perché il cinema, diversamente da altre forme d'arte, non era un'opera per poche persone, ma alla base della sua stessa esistenza vi era il fatto che molte persone dovevano lavorare per la sua produzione per far sì che le masse consumassero il prodotto finale delle riprese, del montaggio e della distribuzione. Così, Anatol Rosenfeld afferma che «nel cinema, l'arte è a servizio della comunicazione. Lo interconnessione di questi elementi è alla base del suo sviluppo e della sua evoluzione perché deriva da una struttura di dimensioni significative proveniente dalla produzione su larga scala» 23 .

La curiosità del pubblico nei confronti delle novità portò velocemente al perfezionamento dei macchinari utilizzati per le riprese, della performance degli attori, richiese la costruzione di scenografie, la creazione di case di produzione ed anche l'impiego di tempo dedicato alla commercializzazione e alla proiezione delle pellicole. $\mathrm{E}$ nonostante le prime proiezioni cinematografiche avessero avuto luogo in Francia, la produzione industriale dei film prosperò più velocemente dall'altra parte del globo terrestre.

20 SINGER, Ben, Modernidade, hiperestímulo e início do sensacionalismo popular, in CHARNEY, Leo, SCHWARTZ, Vanessa R. (org.), O cinema e a invenção da vida moderna, São Paulo, Cosac \& Naify, 2004, p. 112.

${ }^{21}$ BENAJMIN, Walter, L'opera d'arte nell'epoca della sua riproducibilità tecnica, Torino, Einaudi, 2000.

${ }_{22}^{2}$ KRACAUER, Siegfried, La massa come ornamento, Napoli, Prismi, 1982.

${ }^{23}$ ROSENFELD, Anatol, Cinema: arte \& indústria, São Paulo, Editora Perspectiva, 2002, pp. 13-14. 
Già all'inizio del Ventesimo secolo gli Stati Uniti svettavano come mercato in termini di produzione e consumo dei primi film. Agenzie di distribuzione su scala nazionale o regionale nacquero nel 1902 negli Stati Uniti, tra il1904 e 1905 in Francia e 1907 in Germania. Secondo Carlos Augusto Calil24, le prime case di produzione nordamericane si stabilirono inizialmente a New York e Chicago; soltanto nel 1908 il primo produttore si spostò ad Hollywood. Il colonnello William Seling, infatti, tentava di scappare del brutto tempo, dal momento che le riprese erano dipendenti dalla luce solare. Un anno dopo la New York Motion Pictures Co. ebbe la stessa idea. Aldilà della luce naturale, cercavano di scappare dalla vigilanza e dalle richieste di permesso dalla Motion Picture Patents Co. di Thomas Edison (1847-1931). In questo modo, poco per volta, le case produttrici si trasferirono in California.

Hollywood si trasformava così nella Mecca del cinema nordamericano, in grado di concorrere con i film europei. Con investimenti e guadagni maggiori, i produttori e distributori di film nordamericani acquisirono il vantaggio necessario per il perfezionamento tecnico e la professionalizzazione di questo comparto. Il Brasile importava pellicole già dai primi anni del XX secolo e la situazione non ha cambiò tra la fine degli anni Trenta e l'inizio dei Quaranta.

I riferimenti alle grandi produttrici nordamericane comparivano ogni quotidianamente sui giornali del Sergipe durante la Seconda guerra mondiale. I nomi Paramount, United, RKO, Metro e 20 th Century Fox erano costantemente citati per attirare l'attenzione del pubblico, che poteva così associare i film all'industria e all'estetica nordamericana. Secondo Calil, le grandi case di produzione nordamericane erano organizzate verticalmente, «cioè, controllavano gli affari del cinema dalla produzione fino all'esibizione nelle sale apposite o collegate»25. In questa logica il nome dello studio fungeva da vetrina "per un marchio già identificato dal pubblico» ${ }^{26}$.

Gli annunci invitavano le persone a guardare gli spettacoli che venivano proiettati in ogni "cineteatro". La programmazione prevedeva anche più di un film. Il 30 luglio 1940 il "cineteatro" Rex presentava «una colossale combinazione di due superbi film, dal momento che il primo sarà la superproduzione di RKO Radio "Heróis sem Glória" 27 con Sally Elleres, John Bell ed altri, e il secondo sarà la straordinaria pellicola “Sensação no Circo" »28.

24 CALIL, Carlos Augusto, Cinema e Indústria, in XAVIER, Ismail (org), O cinema no século, Rio de Janeiro, Imago Ed., 1996, pp. 45-70.

25 Ibidem, p. 52.

${ }^{26}$ Ibidem.

27 LANDERS, Lew, Danger Patrol, RKO Radio, Stati Uniti, 1937, 59'.

${ }^{28}$ Correio de Aracaju, 30 luglio 1940, p. 2. 
Tra 1939 e 1945 le produzioni hollywoodiane esplorarono le possibilità che il conflitto mondiale offriva agli studios. La guerra aveva portato alla realizzazione di scenaggiature dove si avviluppavano trame di romanzo, azione, dramma, commedia; anche gli studi Disney cercarono ispirazione in figure come quelle Hitler, Himmler e Goering per divertire ed insegnare i bambini attraverso i cartoni animati.

Successivamente all'agosto 1942, il Brasile fu direttamente coinvolto nel conflitto, dichiarando guerra alla Germania e all'Italia. Dopo i siluramenti delle navi brasiliane lungo la costa tra gli Stati del Sergipe e di Bahia, il posizionamento internazionale del paese genererà mutamenti anche della censura dei film prodotti fuori dal Brasile. I film europei, che già erano pochi, praticamente scomparirono dagli schermi brasiliani, mentre i nordamericani continuavano a dominare le sale di proiezione, con la differenza che dopo questo periodo non vi era più problema nel presentare discorsi sfavorevoli nei confronti della Germania, dell'Italia, del Giappone e dei loro governanti ${ }^{29}$.

Le critiche nei confronti dei regimi autoritari e contrari alla democrazia erano permesse. Già nel secondo semestre del 1942 si poté notare l'aumento significativo degli annunci dei film in cui l'argomento principale era la Seconda guerra mondiale. Nell'intento di promuovere il sentimento patriottico, ed anche la vendita dei biglietti, alcuni film finirono per essere presentati come "antinazisti". Come, ad esempio, nel caso di O Espia submarinoso; «O Correio de Aracaju» del 14 ottobre 1942 evidenziava come il film riproducesse «appieno l'eroico ed orribile dramma della guerra attuale nei mari!!!»31. I cinema di Aracaju mettono in luce, con il loro funzionamento, il modo in cui le produzioni cinematografiche tenevano informata la popolazione sui problemi che il mondo affrontava.

Proprio in quest'ottica, il giornale «A Cruzada» raccomandava al pubblico il film Ser ou não ser32, che poteva essere considerato come «una satira tremenda degli uomini "integri" della Gestapo e allo stesso tempo esalta quasi allo stesso modo l'espressione del patriottismo dei figli della Polonia. È una commedia che diverte e insegna molto»33. La segnalazione dei film che mostravano l'aspetto quotidiano della guerra non è un elemento difficile da rinvenire sui giornali. Nell'esempio citato il cinema veniva visto come strumento pedagogico.

29 Il Brasile solo dichiara guerra al Giappone nel giugno di 1945.

${ }^{30}$ KORDA, Alexander, ASHER, Irving, The Spy in Black (U-Boat 29), Columbia, Regno Unito, 1939, 82'. Il film in Italia è conosciuto con il titolo La spia in nero.

${ }^{31}$ Correio de Aracaju, 14 ottobre 1942, p. 3.

${ }^{32}$ LUBITSCH, Ernst, To Be or Not to Be, United Artists, Stati Uniti, 1942, 99'. Il film è conosciuto in Italia con il titolo Vogliamo vivere!

33 A Cruzada, 23 aprile 1943, p. 4. 
L'argomento della guerra compare anche nella sinossi del film Um louco entre loucos 34 , proiettato ad Aracaju nel 1943 e che veniva definito come «la commedia più stupida dell'anno! Semplicemente infernale! Cade dal cielo scappando dagli "Assi"...adotta la sua tattica... ed entrambi lasciano in mutande un colonnello nazista! Non lasciatevi sfuggire l'opportunità di andare a vedere questo sul film "Um louco entre loucos"»35. Il riferimento agli «Assi», «tattica» e «colonnello nazista» sono il segno della presenza degli argomenti e vocaboli dell'epoca del conflitto.

La recensioni dei film venivano inviate dai cinema per poter essere pubblicate sui giornali di Aracaju e attirare così il pubblico nelle sale di proiezione. Il film Nas assas da Glória $^{36}$ sarebbe stato proiettato presso il Cinema Vitoria e la censura sottolineava che «La trama di questo film sviluppa nell'ambiente di una base di addestramento in prossimità di Panama. Il film ha passaggi emozionati, ma può essere visto da tutti, tranne i bambini»37. Ancora una volta il tema della guerra veniva preso in esame, questa volta con riferimento all'addestramento aereo nelle vicine basi di Panama.

Titoli come Lanceiros da Índias8 (proiettato ad Aracaju nel 1944), Gibraltar39 (proiettato nel 1942), Intriga da China (mostrato nel 1939) presentavano la guerra ed il mondo. I paesi erano venivano ritratti nel pieno della guerra, in situazioni che riguardavano pratiche di spionaggio oppure le attività degli eroici soldati nordamericani che avevano lasciato le comodità della loro casa per ristabilire la pace e la civiltà, a bordo di un sottomarino o di un aereo.

Lanceiros da Índia, ad esempio, fu annunciato sul giornale «A Cruzada» del 17 settembre di 1944 come una produzione che avrebbe mostrato agli spettatori «l'India e tutta la sua selvaggia ed esotica bellezza!» ed anche «La magnifica pompa dei palazzi dei raja e la magia della natura selvatica! E, in questo scenario, l'eroismo di alcuni soldati dediti alla lotta per il progresso e per la civilizzazione!»40. In questo caso i soldati non solo si battevano contro la distruzione causata dalle potenze dell'Asse, ma anche contro l'arretratezza derivante dalla mancanza di civilizzazione.

L'analisi della ricezione dei film hollywoodiani da parte degli aracajuani durante la Seconda guerra mondiale deve tenere conto delle condizioni di proiezione delle pellicole, del pubblico che frequentava i cinema e dell'interazione delle persone

\footnotetext{
${ }^{34}$ SCHULBERG, Percival B., The Wife Takes a Flyer, Columbia Pictures, Stati Uniti, 1942, 86'. 35 Ibidem.

${ }^{36}$ BRISKIN, Irwing, Canal Zone, Columbia Pictures, Stati Uniti, 1942, 79'.

37 Ibidem.

38 HATHAWAY, Henry, The Lives of a Bengal Lancer, Paramount Pictures, Stati Uniti, 1935, 109'. Il film è noto in Italia come I lancieri del Bengala.

39 RABINOVITCH, Gregor, Gibraltar, Ciné-Alliance, Francia, 1938, 105’. Il film è conosciuto in Italia come Allarme a Gibilterra.

40 A Cruzada, 17 settembre 1943, p. 3.
} 
all'interno dei cinema della capitale di Sergipe. Indagando sulle pratiche di lettura, Roger Chatier ricorda che «la lettura è sempre una pratica incarnata nei gesti, negli spazi, nelle abitudini»41. In questo senso, anche la pratica della fruizione dei film ad Aracaju era ascrivibile ad una materialità che dev'essere presa in considerazione. I problemi che si verificavano nei cinema furono registrati dai periodici locali e offrono tracce sulla modalità di fruizione del film nella capitale sergipana.

I periodici registrarono vari episodi in cui i frequentatori s'indignarono per ciò che accadeva nei cinema. Quando c'era qualche problema durante la riproduzione delle pellicole, ad esempio, il pubblico protestava. Il giornale «O Nordeste» del 25 febbraio 1939 conteneva una nota che esemplificava il disappunto degli aracajuani di fronte alle difficoltà tecniche sorte nella sala di proiezione. Le proteste si indirizzavano contro «il malcostume, contro la mancanza di educazione di coloro che, quando si trovavano di fronte a un nastro guasto, senza cerimonie, vi fossero o no delle famiglie intente ad assistere alla proiezione, facevano un frastuono assordante. Ieri, abbiamo visto questa situaizone, al cine Rex»42.

Le denunce del pubblico toccavano anche la precarietà del macchinari utilizzato per riprodurre le pellicole. Questo, del resto era un tema che attirava l'attenzione degli habitués dei cinema locali. L'8 luglio 1940 il cinema São Francisco annunciava la proiezione di O Prisioneiro de Zenda43,produzione della United con «scene eccitanti che catturano l'attenzione come pochi film». Oltre ad annunciare i nomi degli attori «Ronald Colman, Madaleine Carolle Douglas Fairbanks Jr.», il cinema prometteva che si trattava di una «copia assolutamente nuova e completa»44, al termine sarebbe stata presentata la serie Deusa de Joba45.

Le denunce dei frequentatori abituali ricadevano anche sui gruppi che si recavano nei locali. I cinema accoglievano tutte le classi e si trasformavano in luoghi per la manifestazione di atteggiamenti non solo apologetici, ma anche contrari all'ordine vigente. Nel buio del cinema, la popolazione si divertiva, imparava e si svelava. Una prova di ciò è che l'esecuzione dell'inno nazionale non sempre era rispettata.

Quando le luci si spegnevano, operai, commercianti, uomini e donne diventavano anonimi. E neppure i poliziotti o i dipendenti del DIP riuscivano ad imporne il rispetto. In questi momenti non era raro che si sentisse «un rumore assordante, originato da

${ }^{41}$ CHARTIER, Roger, «O Mundo como Representação», in Estudos Avançados, 11 5/1991, pp. 173-191. La citazione proviene da p. 178.

42 O Nordeste, 25 febbraio 1939, p. 1.

43 SELZNICK, David O., The Prisoner of Zenda, United Artists, Stati Uniti, 1937, 101'. Il titolo del film in Italia è Il prigioniero di Zenda.

${ }_{44}$ Correio de Aracaju, 8 luglio 1940, p. 2.

45 LEVINE, Nat, Darkest Africa, Republic Pictures, Stati Uniti, 1937, 269’. 
urle, scalpiccio e fischi [...] durante tutto il tempo dell'esecuzione dell'inno»46. Anche il fatto che alcuni rimanessero con i propri cappelli in testa non era gradito agli spettatori più conservatori.

I progetti che avevano l'obiettivo di omologare la visione di mondo dei brasiliani, non ottenevano risultati omogenei. In merito a questa diversità, Jacques Revel47 raccomanda agli storici di prendere in considerazione il fatto che le società sono gerarchizzate e non ugualitarie, e da questa situazione deriva il fatto che le società siano così complesse, permeata di rapporti tra il forte ed il debole. La possibilità di trovare lo stesso soggetto in contesti sociali diversi permette la costruzione di una storia costruita completametne a partire dal basso. Revel evidenzia le possibilità che si aprono con l'approccio del soggetto alla società.

In parte questa situazione era propiziata dalla distribuzione degli spazi, del tutto peculiare nei "cineteatri". C'erano prezzi diversi per i posti: in platea o in galleria, in cui biglietto era più economico. Dal momento che i posti della galleria si trovavano sopra gli altri, era possibile fare confusione senza farsi vedere. Le denunce del comportamento di coloro che acquistavano i biglietti popolari erano frequenti. Il problema «è che "la galleria" parla ad alta voce, e che alcuni di loro, che già hanno visto il film, raccontano ciò che accadrà: "la ragazza cadrà", "lui morirà", "dopo si sposeranno" ed altre cose che levano il piacere della sorpresa e dell'attesa in chi guarda» 48 , denunciava un frequentatore dei cinema.

Vi era un conflitto tra quelli che compravano i posti in platea e quelli che lo acquistavano in galleria. La conflittualità emergeva dai giornali. Molto spesso, coloro che acquistavanoposti in platea si lamentavano di chi andava galleria, perché questi ultimi pagavano meno di loro per entrare nei cinema. Nel cinema Guarany, ad esempio, durante la rappresentazione matinê, la platea costava Reais 1.500, mentre l'ingresso la galleria Reais 80049; già la soirée costava di più: in platea Reais 3.500, l'entrata a spettacolo iniziato Reais 1.700 e la galleria Reais 1.100.

Accompagnati da recensioni ufficiali e grazie all'entusiasmo nei confronti delle produzioni hollywoodiane, i cinema presentavano la guerra nell'ottica nordamericana. Però, ad Aracaju, le sale di proiezione erano anche luoghi in cui dare dimostrazione dell'ingresso nella modernità, anche se ciò non accadeva sempre.

46 O Nordeste, 26 settembre 1939, p.1

47 REVEL, Jacques, Giochi di scala: la microstoria alla prova dell'esperienza, Roma, Viella, 2006.

${ }^{48}$ Correio de Aracaju, 4 dicembre 1939, p. 1.

${ }^{49}$ Folha da manhã , 3 gennaio 1939, p. 1. 
Nell'epoca in cui si sperava di instillare l'ordine e l'obbedienza delle norme, alcuni aracajuani utilizzavano il buio del cinema per abusare della loro libertà. La mancanza di rispetto nell'esecuzione dell'inno nazionale, le battute pronunciate durante gli spettacoli ed anche l'abitudine di alcuni di assistere ai film più di una volta per annunciarne la trama in anticipo, irritava parte degli spettatori, scocciati dal dover condividere lo stesso spazio con certe persone.

Ma la confusione aveva anche una sua logica di funzionamento. La confusione si verificava solo a luci spente. I tentativi di controllare la condotta degli spettatori, in ossequio alla legislazione emanata dall'Estado Novo, si scontrava con le tatticheso adottate dalla popolazione per sottrarsi all'intimidazione creata dalla presenza della polizia nelle sale di cinema. Oltre a prendere in esame questi artifici sociali del quotidiano, Michel de Certeau ${ }^{1}$ evidenzia come il quotidiano sia segnato da scontri tra i forti, che determinano le condizioni dei contrasti sociali, e i deboli, che cercano di ottenere vantaggi in certe situazioni resistendo all'ordine imposto.

Secondo Alexandre Busko Valim52, gli studi sul rapporto tra storia e cinema hanno suggerito la necessità di studiare la produzione, la mediazione e la ricezione dei film in modo integrato. Questo in modo da poter considerare importante non solo l'analisi della produzione di significato dei film, ma anche le condizioni in cui questo film è realizzato, i mezzi con cui è entrato in circolazione, le sue forme di promozione, di riproduzione e di ricezione.

La ricezione è definita come il punto più irraggiungibile dell'analisi del rapporto tra storia e cinema. Valim evidenzia come i film siano fonti importanti per lo studio delle rappresentazioni, ma non dicano molto sul pubblico o sulle condizioni di produzione, distribuzione e riproduzione. Negli ultimi venti anni si è preso in considerazione la proiezione considerandola come aspetto importante. L'autore afferma che gli studi «rivelano come le differenze tra le pratiche di riproduzione tra distinte città o piccole comunità rendano complesso il rapporto tra l'azione di andare al cinema ed altre pratiche sociali»53. Si propone, allora, una analisi più complessa,che consideri altri aspetti del rapporto tra storia e cinema.

La Seconda guerra mondiale era divenuta il tema di vari film, ma anche in questo campo il conflitto interferiva nella politica e negli affari esteri brasiliani. La rottura dei

\footnotetext{
50 Sul concetto di tattica, si veda: De CERTEAU, Michel, L’invenzione del quotidiano, Roma, Edizioni Lavoro, 2001.

51 Ibidem.

52 VALIM, Alexandre Busko, História e Cinema, in CARDOSO, Ciro Flamarion, VAINFAS, Ronaldo, Novos Domínios da História, Rio de Janeiro, Elsevier, 2012, pp. 283-300.

53 Ibidem, p. 288.
} 
rapporti diplomatici con i paesi dell'Asse permise l'ingresso di film nordamericani, che erano latori di un discorso più tendenzioso in merito al confronto bellico. Solo dopo i siluramenti dell'agosto del 1942 e l'entrata in guerra del Brasile a fianco degli alleati alcuni film, ad esempio, O grande ditador54, poterono essere mostrati nel paese.

I cinema aracajuani durante la Seconda guerra mondiale erano dai film nordamericani prodotti a Hollywood. Questi avevano la capacità di portare a conoscenza del pubblico le ultime novità che circolavano nel mondo, facendo ricorso a risorse tecniche differenti - come il film a colori o doppiato in portoghese - o grazie agli attori più famosi o parlando degli argomenti più attuali, come avveniva con i film di guerra. Accompagnati di recensioni ufficiali e grazie all'infatuazione nei confronti delle produzioni hollywoodiane, i cinema presentavano la guerra attraverso la prospettiva nordamericana.

In questo modo questi film spiegavano gli avvenimenti, le ragioni del conflitto, i nuovi mezzi bellici impiegati, gli effetti della distruzione di una guerra di simili proporzioni e i sentimenti che spingevano gli uomini a lottare contro la malvagità del nemico. La narrazione cinematografica nordamericana trovava risonanza tra il pubblico che frequentava i cinema aracajuani. Ed il governo federale appoggiava l'idea che tutti dovrebbero unirsi per combattere i regimi autoritari ed i nemici della democrazia fuori del Brasile.

54 CHAPLIN, Charlie, The Great Dictator, United Artist, Stati Uniti, 1940, 125'. Il titolo italiano del film è Il grande dittatore. 


\section{* L'autore}

Andreza S. C. Maynard si è addottorata in Storia presso l'Universidade Estadual Paulista (UNESP) ed è attualmente post-dottoranda presso la Universidade Federal do Rio de Janeiro (UFRJ). È membro del Grupo de estudo do tempo presente (GET) dell'Universidade Federal de Sergipe.

URL: < http://www.studistorici.com/progett/autori/\#SantosCruzMaynard >

\section{Per citare questo articolo:}

SANTOS CRUZ MAYNARD, Andreza, «La Seconda guerra mondiale sugli schermi: una analisi del cinema nel Nordeste brasiliano (1939-1945)», Diacronie. Studi di Storia Contemporanea : Periferie. Cultura, economia, politica, 29/3/2014,

URL: < http://www.studistorici.com/2014/3/29/SantosCruzMaynard_numero_17/ >

Diacronie Studi di Storia Contemporanea $\beta$ www.diacronie.it

Risorsa digitale indipendente a carattere storiografico. Uscita trimestrale.

redazione.diacronie@hotmail.it

Comitato di redazione: Jacopo Bassi - Luca Bufarale - Elisa Grandi - Deborah Paci - Fausto Pietrancosta - Matteo Tomasoni - Luca Zuccolo 